\title{
不同土壤水分条件下洪泛平原湿地披芨草叶片厚 度与叶脉性状的关系
}

\author{
韩 玲 赵成章* 徐 婷 冯 威 段贝贝 \\ 西北师范大学地理与环境科学学院, 甘肃省湿地资源保护与产业发展工程研究中心, 兰州 730070
}

\begin{abstract}
摘 要 叶片厚度和叶脉性状的关联性影响着叶片水分的动态平衡, 对揭示植物叶片水分运输与生长速率耦合的生理生态 学机制具有重要的意义。该文选择位于张掖市黑河干流边缘的洪泛平原湿地作为实验地, 以河边为起点, 沿垂直河岸线的方 向依次设置I (50.07\%)、II (38.77\%)、III (31.5\%)、IV (20.4\%) 4个土壤含水量梯度样地, 采用标准化主轴估计(SMA)方法, 以 叶脉密度和叶脉直径分别表示叶脉性状, 研究了不同土壤水分条件下芨芨草(Achnatherum splendens)种群的叶片厚度与叶脉 性状的生长关系。结果表明: 随着湿地群落土壤含水量的逐渐降低, 湿地群落的植被密度和高度逐渐降低, 土壤电导率逐渐 增大; 芨芨草的叶脉密度、叶片厚度、水分利用效率和光合有效辐射呈逐渐增加的趋势, 叶脉直径、蒸腾速率和分枝数呈逐 渐减小的趋势, 净光合速率和株高呈先增大后减小的趋势; 随着湿地群落土壤含水量的逐渐降低, 芨芨草叶片厚度与叶脉密 度、叶脉直径的相关性在不同样地间存在差异; 芨芨草叶片厚度与叶脉密度呈显著的正相关关系, SMA的斜率呈逐渐减小的 趋势, 且在样地I和样地IV与1.0存在显著差异; 叶片厚度与叶脉直径呈显著的负相关关系, SMA的斜率呈逐渐增大的趋势, 且 在样地 $\mathrm{I}$ 和样地 $\mathrm{IV}$ 与- 1.0 存在显著差异。芨芨草在土壤水分较充足的湿地群落倾向于少量粗脉的薄叶片构建方式, 在受土壤水 分胁迫时选择大量细脉的厚叶片的生长模式, 体现了湿地植物在资源异质性分布的生境中根据其功能需求在自身性状之间 进行的资源优化配置。
\end{abstract}

关键词 叶片厚度; 叶脉密度; 叶脉直径; 异速生长; 芨芨草; 洪泛平原湿地

引用格式: 韩玲, 赵成章, 徐婷, 冯威, 段贝贝 (2017). 不同土壤水分条件下洪泛平原湿地芨菆草叶片厚度与叶脉性状的关系. 植物生态学报, 41 , 529-538. doi: 10.17521/cjpe.2016.0123

\section{Relationships between leaf thickness and vein traits of Achnatherum splendens under different soil moisture conditions in a flood plain wetland, Heihe River, China}

HAN Ling, ZHAO Cheng-Zhang ${ }^{*}$, XU Ting, FENG Wei, and DUAN Bei-Bei

College of Geography and Environmental Science, Northwest Normal University, Research Center of Wetland Resources Protection and Industrial Development Engineering of Gansu Province, Lanzhou 730070, China

\section{Abstract}

Aims The correlations between leaf thickness and vein traits influenced the leaf hydraulic dynamic balance, and there were important meanings to reveal ecophysiological mechanisms of plant leaves water transport and growth rate. Our objective was to study the changes in the relationship between leaf thickness and vein traits (vein diameter, vein density) of Achnatherum splendens populations by using standardized major axis estimation (SMA) method under different soil moisture conditions located in flood plain wetland of Zhangye.

Methods The study site was located at flood plains wetland of Zhangye, Gansu Province, China. Selecting a starting point along the vertical direction of the river, in turn, along the soil moisture gradient, four plots were set up at intervals of $40 \mathrm{~m}$, plot I (50.07\%), plot II (38.77\%), plot III (31.5\%) and plot IV (20.4\%). From each of the four sample plots, seven samples were collected, resulting in $(5 \mathrm{~m} \times 5 \mathrm{~m})$ a total of 28 samples. Community traits (height, density) and soil physical and chemical properties were investigated. Six individual samplings of $A$. splendens from each plot were used to measure the leaf thickness, vein density and vein diameter in laboratory. In addition, the photosynthetically active radiation $(P A R)$, leaf net photosynthetic rate $\left(P_{\mathrm{n}}\right)$ and transpiration rate $\left(T_{\mathrm{r}}\right)$ of A. splendens were measured in natural environment. The 28 plots were categorized into groups I, II, III and IV, and SMA estimation method was then used to examine the allometric relationship among leaf thickness, vein density and vein diameter.

收稿日期Received: 2016-04-05 接受日期Accepted: 2017-02-28

* 通信作者Author for correspondence (E-mail: zhaocz601@163.com) 
Important findings With a decreased soil moisture, the plant density and height displayed a pattern of steadily declining, while the soil electrical conductivity increased, In addition, the vein density, leaf thickness, water use efficiency (WUE), PAR and twig number of A. splendens displayed a pattern of initial decrease, whereas the vein diameter and $T_{\mathrm{r}}$ increase gradually, $P_{\mathrm{n}}$ and plant high displayed changing trends of increasing-decreasing. The leaf thickness was negatively associated with the vein density, vein diameter, and the relationship varied with the soil moisture conditions $(p<0.05)$. There was a significant positive relationship $(p<0.05)$ between the leaf thickness and vein density. The SMA slope of the regression equation gradually decreased and was significantly different from $1.0(p<0.05)$ on plot I and IV. In addition, along decreased soil moisture, the standardized major axis slope of regression equation in the scaling relationships between the leaf thickness and vein diameter gradually increased and was significantly different from $-1.0(p<0.05)$ on plot I and IV.

Key words leaf thickness; vein density; vein diameter; allometry; Achnatherum splendens; flood plain wetland

Citation: Han L, Zhao CZ, Xu T, Feng W, Duan BB (2017). Relationships between leaf thickness and vein traits of Achnatherum splendens under different soil moisture conditions in a flood plain wetland, Heihe River, China. Chinese Journal of Plant Ecology, 41, 529-538. doi: 10.17521/cjpe.2016.0123

植物叶片的水分输导及动态平衡是SPAC系统 中水分迁移的一个重要环节, 不仅受植物本身的调 节和控制, 而且还受外界环境条件的影响(张志亮等, 2014), 水流通过直径递减的一系列叶脉从维管束 木质部末端进入表皮细胞和气孔下腔附近的蒸发部 位, 叶片中的水分运输路径及效率对整个植物体的 叶片几何构型构建、水力特性及资源的获取与权衡 利用效率等具有较大的影响 (Sack \& Scoffoni, 2013)。叶片功能性状作为联系植物生理适应性和环 境梯度间的桥梁, 叶片厚度影响着叶片的水分供 应、存储以及光合作用中物质和能量的交换过程 (Scoffoni et al., 2011; Ogburn \& Edwards, 2012), 叶脉 密度和叶脉直径可反映叶脉系统的水分、养分和光 合产物等物质运输的效率和安全性以及抵抗外界干 扰的能力(Brodribb \& Jordan, 2011; Sack \& Scoffoni, 2013)。面对资源异质性分布的生境和植物生活史中 光合产物的供需矛盾, 叶片各功能性状的自我调整 与适应显得至关重要(许洺山等, 2015), 叶片厚度的 资源投入有利于减少水分的蒸腾散失并提高固碳能 力(Gago et al., 2014; 全先奎和王传宽, 2015); 较粗 直径导管的高水分流动速率提高了叶脉水分输导的 “有效性”, 却降低了水分运输中的“安全性” (Givnish, 1987; Matthew-Ogburn \& Edwards, 2013), 而增 加叶脉密度能够增加叶片水分供应的面积和路径, 使得水分运输的安全性得到保障(Nardini et al., 2010), 三者的资源经济权衡机制与协同进化策略 共同对叶片水分供应与散失的平衡、植物生理活动 的能量供给和生存适应方面具有不可替代的调控作 用, 体现了叶脉和叶性状间存在着蒸腾成本和光合 收益的匹配和权衡关系(Sack et al., 2013)。干旱区土
壤水分格局作为影响植物水分来源及利用策略的关 键因子, 与光照和生存空间等多重环境变化及选择 压力的交互作用对植物性状的资源获取方式和生理 活性的表达具有直接的限制作用(Pausas \& Austin, 2001)。土壤水分亏缺会限制植物固碳功能的发挥及 引起植物体木质部空穴化和栓塞现象的产生, 进而 影响叶片的气体交换和植物的正常生长发育(李荣 等, 2015; 许洺山等, 2015)。叶片厚度和叶脉结构等 功能性状能够敏锐地感应叶片的输水阻力对生理功 能的阻碍(Scoffoni et al., 2015), 为有效地加强植物 体内的水分运输与利用效率和进一步增强现实竞争 力, 植物在叶片的叶肉组织和叶脉网络系统的构造 上进行权衡投资(Wilson et al., 1999; Reich \& Cornelissen, 2014), 促使植物调整叶脉结构和叶片厚度 的生物量分配和叶片构型构建, 从而提高植物对土 壤水分的有效利用(Moles et al., 2014; 王瑞丽等, 2015), 实现叶片生理特性和环境空间异质性的精 细协调, 体现了植物最经济的生境适应性和表型可 塑性机制(Silvertown \& Charlesworth, 2001), 即植物 在不同功能和性状之间对有限资源的分配存在着互 相诨制的权衡作用(Nardini \& Luglio, 2014)。因此, 研究湿地群落芨苃草叶片厚度和叶脉性状对不同土 壤水分梯度的响应规律, 可以探索湿地植物碳水代 谢关系和高效用水的生理生态学机制。

芨菆草(Achnatherum splendens) 是我国西北地 区常见的多年生密从性草本植物, 草秆坚硬、直立 丛生、根系发达、须根较多, 叶片拥有较强的叶脉 机械支撑能力和水分输导组织, 芨芨草并非喜盐植 物, 与典型盐生植物相比, 仅是生态幅较宽的耐盐 旱生物种, 通常发育于轻度盐化的河湖边缘以及低

www.plant-ecology.com 
洼地等隐域性生境, 是一种优良的饲用植物、纤维 植物和水土保持植物(张明娟等, 2012; 韩玲等, 2016)。近年来, 叶脉功能性状与叶片厚度(Scoffoni et al., 2011)、叶大小(Scoffoni et al., 2015; 徐婷等, 2016)的异速生长关系、叶脉网络性状与叶片水力特 性(Blonder et al., 2010; Zhang et al., 2012)、叶片碳构 建(Reich \& Cornelissen, 2014)的关联性, 以及植物 输水结构与耐旱性的关系(李荣等, 2015)、叶片功能 属性沿水分和光照梯度的变化趋势 (Domínguez et al., 2012; Perez-Ramos et al., 2012)等方面已进行了 大量的研究, 解释了植物适应特定环境的生理生态 学机制; 芨芨草的水分利用策略(Wang et al., 2004) 等也受到了关注, 但芨芨草叶片形态和叶脉性状的 异速生长规律研究不深入, 尤其是湿地群落不同土 壤水分生境下叶片厚度与叶脉密度、叶脉直径的生 长关系对植物生理功能和异质性资源分布的响应机 理尚不清晰。鉴于此, 本文以张掖湿地芨芨草种群 为研究对象, 探讨张掖湿地群落不同土壤水分梯度 下芨芨草叶片厚度-叶脉密度, 叶片厚度-叶脉直径 的相互关系及变化规律, 旨在理解不同环境下植物 叶片的资源权衡分配和物理构建方式, 有助于揭示 湿地植物为获得最大光合生产力所形成的水资源利 用策略和生理生态适应性。

\section{1 材料和方法}

\section{1 研究区域和样地概况}

研究区位于甘肃省张掖市甘州区西城驿黑河 干流河床边缘缓坡地带的洪泛平原湿地, 地理位置 为 $38.56^{\circ} \mathrm{N}, 100.26^{\circ} \mathrm{E}$, 海拔 $1482.7 \mathrm{~m}$, 属温带大陆 性气候, 雨热同期, 年平均气温 $7.8{ }^{\circ} \mathrm{C}, \geqslant 0{ }^{\circ} \mathrm{C}$ 积温 为 $2734{ }^{\circ} \mathrm{C}$, 年降水量 $132.6 \mathrm{~mm}$, 主要集中在6-9月, 年蒸发量 $1986.5 \mathrm{~mm}$, 年日照时数 $3077 \mathrm{~h}$, 土壤以 砾石土、灰棕荒漠土和草甸土为主, 湿地内土壤类 型的多样化决定了旱生、盐生、湿生等植物群落的 形成, 植被分布具有明显的水平分异特征, 以湿生 植物、盐生植物与陆生乔灌林木为主。主要植物有 芨菆草、芦苇(Phragmites australis)、冰草(Agropyron cristatum)、节节草(Equisetum ramosissimum)、苦豆 子(Sophora alopecuroides)、宽苞水柏枝(Myricaria bracteata)、多枝柽柳(Tamarix ramosissima)、旱柳 (Salix matsudana)和沙柊(Elaeagnus angustifolia)等。 该研究区属于张掖黑河国家湿地保护区核心区, 不

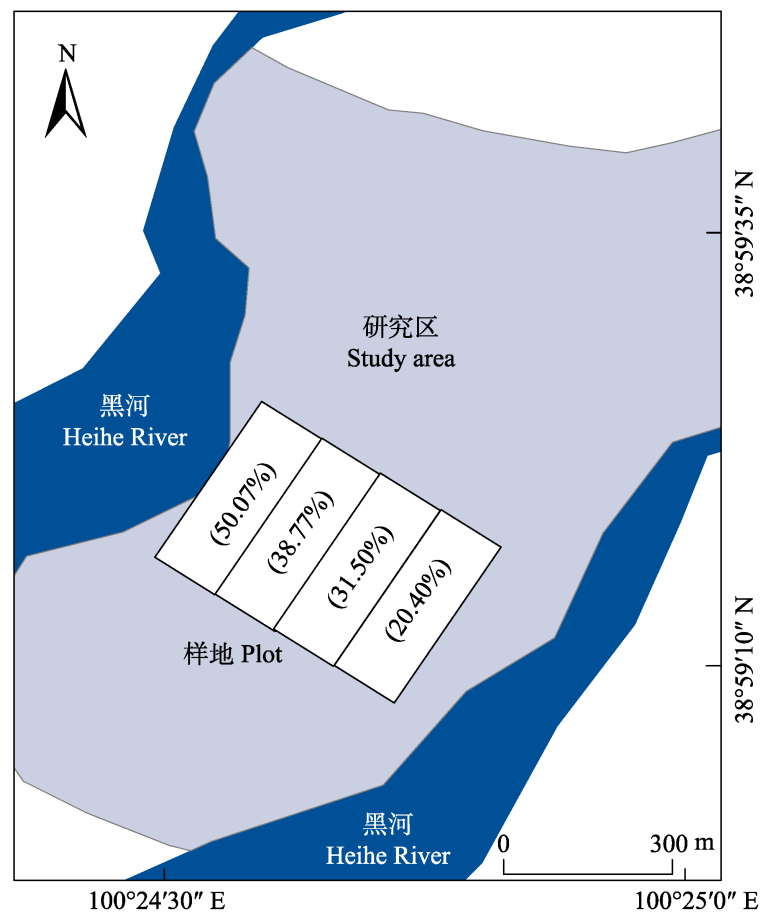

图1 研究区概况和样地设置图。

Fig. 1 Study area and locations of measured plots.

存在家畜放牧和人类活动等干扰因素, 能够真实地 反映土壤水分等自然属性对植物生物学特性和繁殖 等功能的影响(韩玲等, 2016)。

\section{2 实验设置}

黑河干流从蒀落峡进入河西走廊, 河流两岸形 成广阔的倾斜平原。受季节性洪水和地下潜水的影 响, 在河岸边缘发育了大片由芨芨草、芦苇、苦豆 子等草本植物和多枝柽柳、沙苯、旱柳等乔木为主 要植被的洪泛平原湿地。受地下水埋深的影响, 植 被呈现明显的带状分布特征。在实地考察观测的基 础上, 选择一处从河岸边到湿地与荒漠交界处长约 $200 \mathrm{~m}$ 且以菆芨草为优势种的湿地群落进行实验。 首先, 进行地下水埋深的测量(在每条样带上从近 河岸开始每间隔 $10 \mathrm{~m}$ 用铁铲挖至地下水流出, 待水 位稳定后测量地下水埋深), 然后根据地下水埋深 变化规律，从黑河沿岸以垂直于河岸线的方向间隔 $40 \mathrm{~m}$ 依次设置 4 条长 $20 \mathrm{~m}$ 、宽 $150 \mathrm{~m}$ 的平行样带I、II、 III和IV, 分别为: 样地 I, 地下水埋深 $0.5-1.0 \mathrm{~m}$, 该 样地受季节性和间歇性洪水的影响, 地表被水面半 永久性覆盖, 其中11-4月份覆盖, 5-10月份不覆盖, 地下水位处在地表或附近, 主要植物有芨芨草、芦 苇、宽苍水柏枝和多枝柽柳等; 样地II, 地下水埋深 1.0-1.5 m, 该地段一般只是间歇性淹水，4-11月份 
地表偶尔被覆盖, 地表水在一年中出现时间较短, 主要植物有芨芨草、苦豆子、旱柳等; 样地III, 地 下水埋深1.5-2.0 m, 该地段一般不会淹水, 植被主 要依靠地下潜水生存, 主要植物有芨芨草、节节草、 沙朶等; 样地IV, 地下水埋深2.0-2.5 m, 土壤受河 流水位影响极小, 土壤水分不能满足大多数植物的 生长, 主要植物有芨菆草、冰草等。

2015年8月初, 于基本无明显降水、土壤水盐状 况相对稳定的期间进行取样。在选定的4个样地中各 随机选取 7 个 $5 \mathrm{~m} \times 5 \mathrm{~m}$ 的样方, 共计 28 个样方。首先 分别测定每个样方内湿地群落所有植物的高度和密 度, 芨芨草以丛为单位; 然后在每个小样方随机挖 掘 $1 \mathrm{~m} \times 1 \mathrm{~m} \times 0.5 \mathrm{~m}$ 的土壤剖面, 用环刀 $\left(200 \mathrm{~cm}^{3}\right)$ 分5层间隔 $10 \mathrm{~cm}$ 采取土样, 重复 3 次, 现场编号、各 土层均匀混合称鲜质量, 然后带回实验室在 $105{ }^{\circ} \mathrm{C}$ 的烘箱内烘干 $12 \mathrm{~h}$, 取出称质量, 计算出各样地 0-50 cm土层土壤质量含水量(韩玲等, 2016); 最后 在每一个样方选择 6 从芨芨草(株高为大中小各 2 丛), 用卷尺测量从基部到最高分枝的高度, 定为株高, 并记录菆芨草株从分枝数, 然后选取每个株从最外 层4个方位充分伸展且健康完整的 $2-3$ 个叶片, 做好 标记后进行以下步骤:

(1)植物光合参数测定。于 8 月 $15-18$ 日(代表植 物生长旺盛期), 选择晴朗天气的9:00-12:00进行气 体交换参数的测定。光合测定使用GFS-3000便携式 光合测量系统(Heinz Walz GmbH, Bavaria, Germany), 测量过程中使用人工红蓝光源, 光合有效辐射 $(P A R)$ 为 $1200 \mu \mathrm{mol} \cdot \mathrm{m}^{-2} \cdot \mathrm{s}^{-1}, \mathrm{CO}_{2}$ 浓度约为 $340 \mu \mathrm{mol} \cdot \mathrm{mol}^{-1}$, 相对湿度 $(R H)$ 保持在 $40 \%-50 \%$, 流速设定为 $750 \mu \mathrm{mol} \cdot \mathrm{s}^{-1}$, 叶室面积为 $8 \mathrm{~cm}^{2}$, 对做好标记的每 个叶片记录 5 组数据用于统计分析, 分别测定叶片 的净光合速率 $\left(P_{\mathrm{n}}\right)$ 、蒸腾速率 $\left(T_{\mathrm{r}}\right)$ 等参数 $($ 任青吉等, 2015), 对于不能充满叶室的叶片, 则保存于湿润的 封口袋中, 带回实验室, 用便携式激光叶面积仪 (CI-202, Walz, Camas, USA)测定叶面积, 进而计算 $P_{\mathrm{n}}$ 和 $T_{\mathrm{r}}$, 水分利用效率 $(W U E)=P_{\mathrm{n}} / T_{\mathrm{r}}$ (韩玲等, 2016)。

(2)光合有效辐射测定。于 8 月 15-18日用手持光 量子计(3415F, Walz, Plainfield, USA)在9:00-11:00 对 4 个土壤水分梯度内所有供试芨芨草株从进行 $P A R$ 测定, 分别选择植株上方、中层(株高 $1 / 2$ 处)和地 表处向外距灌层 $15 \mathrm{~cm}$ 处测量(韩玲等, 2016)。

(3)叶片厚度和叶脉性状的测定。基于每个芨夰
草被标记的2-3个叶片, 采集后保存于湿润的封口 袋中，带回实验室用福尔马林-乙酸溶液 $37 \%$ 甲醛 溶液，50\%乙醇和 $13 \%$ 冰醋酸溶液)固定(徐婷等, 2016)。(1)先进行叶片厚度(单位: $\mathrm{mm}$ )的测定, 使用 游标卡尺测量(精确度 $0.01 \mathrm{~mm}$ ), 测量时尽量选择叶 片中心部位, 并避开叶脉, 每个叶片重复测量 5 次, 最后取平均值为叶片厚度; (2)叶脉性状的测定, 先 用 $5 \% \mathrm{NaOH}$ 的乙醇进行化学清理、番红-固绿染色 (Berlyn \& Miksche, 1976)、用水包埋呈透明薄膜状, 然后在体视显微镜(SMZ168-BL, Motic, 香港, 中国) 下放大 10 倍后拍照, 每个叶片拍摄 10 个视野用于叶 脉性状的观测, 最后用Motic Images Plus 2.0软件获 得各个照片中的叶脉直径和叶脉的总长度, 所有照 片中得出的平均值即为叶脉直径和叶脉密度; (3)最 后用便携式激光叶面积仪(CI-202, Walz, Camas, USA)对每个叶片进行扫描, 得到叶面积, 叶脉密度 用单位叶面积(单位: $\mathrm{mm}^{2}$ ) 的叶脉总长度(单位: $\mathrm{mm}$ ) 表示(Sack et al., 2012)。

(4)土壤盐分测定。用电导法测定土壤含盐量, 在室温下称取过 $2 \mathrm{~mm}$ 篮的风干土样 $10 \mathrm{~g}$, 加 $50 \mathrm{~mL}$ 去 $\mathrm{CO}_{2}$ 蒸馏水(水土质量比为 $5: 1$ ), 取浸出液, 置振 荡机上振荡 $5 \mathrm{~min}$ 。将布氏漏斗与抽气系统相连后把 悬浊的土浆缓缓倒入漏斗, 直至抽滤完毕, 滤液倒 入三角瓶备用(张雅琼等, 2010)。用上海雷磁仪器厂 生产的DDS-11C便携式电导仪测浸出液的电导率, 3 次重复, 取平均值即为土壤电导率值(韩玲等, 2016)。

\section{3 数据分析}

对湿地群落中 4 个水分梯度中芨芨草叶片厚 度、叶脉密度和叶脉直径的实验数据先进行以 10 为 底的对数转换, 使之符合正态分布后再进行分析。 用于各土壤水分梯度中任意两个叶性状的异速生长 方程的参数估计, 主要采用标准化主轴估计(SMA) 方法(Warton et al., 2006), 采用 $y=a x^{b}$, 线性转换成 $\log (y)=b \log (x)+\log (a)$, 式中 $x$ 和 $y$ 表示两个特征参 数, $a$ 为性状关系的截距, $b$ 为斜率, 即异速生长参数 或相对生长的指数, 当 $|b|=1$ 时, 表示两者是等速变 化关系; 当 $|b|$ 显著偏离 1 时, 两者间为异速变化关 系(Harvey \& Pagel, 1991), 由软件SMATR Version 2.0 (Falster et al., 2012)计算完成, 每一个回归斜率 的置信区间根据Pitman (1939)方法计算, 并采用 Warton和Weber (2002)的方法对每个密度的斜率进 
行异质性测试。不同水分梯度间植物功能性状平均 值的差异比较采用单因素方差分析 $(a=0.05)$ 。实验 所用数据采用SPSS 16.0 软件进行处理分析, 用 Excel软件绘图。

\section{2 结果分析}

\section{1 不同土壤水分条件下湿地群落生物学特征和 土壤理化性质}

不同样地湿地群落的生物学特征、土壤理化性 质和 $P A R$ 的变化如表1所示。不同样地间的湿地群落 的土壤含水量、土壤电导率、群落密度、高度和 $P A R$ 存在显著差异 $(p<0.05)$, 从样地 I到样地 II、样地III 和样地 IV, 土壤含水量呈逐渐减小趋势, 样地IV比 样地减少了 $59.26 \%$; 随着不同样地土壤含水量的 逐渐降低, 湿地群落的平均高度和密度均呈现逐渐 减小的趋势, 样地IV比样地I群落高度减少 $42.42 \%$, 密度降低 $67.69 \% ; P A R$ 和土壤电导率随着土壤含水 量的降低均呈逐渐增加的趋势, 样地IV比样地 I $P A R$ 增加了 1.17 倍, 土壤电导率增加了 2.68 倍。

\section{2 不同土壤水分条件下湿地植物芨芨草各功能} 性状变化

芨芨草各功能性状在不同样地的变化如表2所
示。随着土壤含水量的逐渐降低, 芨芨草的株高和 净光合速率 $\left(P_{\mathrm{n}}\right)$ 呈先增大后减小的趋势, 株高的变 化趋势为样地 III $>\mathrm{II}>\mathrm{I}>\mathrm{IV}, P_{\mathrm{n}}$ 的变化趋势为样地 III $>$ II $>$ IV $>$ I, 二者均在样地III出现较大值, 样地 IV 较样地 $\mathrm{I}, P_{\mathrm{n}}$ 增加了 $5.07 \%$, 株高减小了 $26.6 \%$; 芨 芨草分枝数、叶脉直径和蒸腾速率 $\left(T_{\mathrm{r}}\right)$ 均呈逐渐减小 的趋势，样地IV较样地I分别减少了 $21.15 \%$ 、 $44.26 \%$ 和 $7.03 \%$; 叶片厚度、叶脉密度和水分利用效率 (WUE)均呈逐渐增加的趋势, 从样地 $\mathrm{I}$ 到样地 $\mathrm{IV}$ 分别 增加了 $14.48 \%$ 、23.69\%和 $12.95 \%$; 芨芨草各功能性 状的变化在样地II和样地III之间均无显著差异 $(p>$ 0.05)。表明样地II和样地III的湿地群落生境胁迫较 小, 较适宜湿地群落芨夰草种群的生存。

\section{3 不同土壤水分条件下湿地植物芨芨草叶片厚 度和叶脉密度的关系}

如图2所示, 不同土壤水分条件下芨芨草叶片 厚度和叶脉密度呈显著的正相关生长关系 $(p<$ 0.05)。通过SMA分析得到, 芨芨草在不同样地间的 SMA斜率存在差异, 样地I为1.1964 (95\%的置信区 间: $C I=0.9920-1.163$ 1)、样地II为 $0.9742(95 \%$ 的置 信区间: $C I=0.802$ 2-0.994 3)、样地III为 0.9626 (95\%的置信区间: $C I=0.8854-0.9721$ )、样地IV为

表1 不同样地的湿地群落生物学特征和土壤理化性质(平均值土标准误差, $n=30$ )

Table 1 Wetland community biology characteristics and soil physicochemical properties in different plots (mean $\pm S E, n=30)$

\begin{tabular}{|c|c|c|c|c|c|}
\hline $\begin{array}{l}\text { 样地 } \\
\text { Plot }\end{array}$ & $\begin{array}{l}\text { 土壤含水量 } \\
\text { Soil moisture content }(\%)\end{array}$ & $\begin{array}{l}\text { 土壤电导率 } \\
\text { Soil electrical conductivity }\left(\mathrm{ms} \cdot \mathrm{cm}^{-1}\right)\end{array}$ & $\begin{array}{l}\text { 高度 } \\
\operatorname{High}(\mathrm{cm})\end{array}$ & $\begin{array}{l}\text { 密度 } \\
\text { Density }\left(\text { plant } \cdot \mathrm{m}^{-2}\right)\end{array}$ & $\begin{array}{l}\text { PAR } \\
\left(\mu \mathrm{mol} \cdot \mathrm{m}^{-2} \cdot \mathrm{s}^{-1}\right)\end{array}$ \\
\hline I & $50.07 \pm 1.24^{\mathrm{a}}$ & $3.01 \pm 0.19^{\mathrm{d}}$ & $171.20 \pm 5.24^{\mathrm{a}}$ & $19.5 \pm 0.55^{\mathrm{a}}$ & $645.30 \pm 13.18^{\mathrm{d}}$ \\
\hline II & $38.77 \pm 1.03^{b}$ & $5.88 \pm 0.23^{\mathrm{c}}$ & $146.37 \pm 4.62^{b}$ & $16.4 \pm 0.34^{b}$ & $833.10 \pm 22.48^{c}$ \\
\hline III & $31.50 \pm 1.01^{\mathrm{c}}$ & $7.79 \pm 0.33^{\mathrm{b}}$ & $127.33 \pm 3.25^{\mathrm{c}}$ & $10.3 \pm 0.24^{\mathrm{c}}$ & $921.40 \pm 31.24^{\mathrm{b}}$ \\
\hline IV & $20.40 \pm 0.67^{\mathrm{d}}$ & $11.90 \pm 0.55^{\mathrm{a}}$ & $98.57 \pm 3.66^{\mathrm{d}}$ & $6.3 \pm 0.1^{\mathrm{d}}$ & $1397.10 \pm 45.25^{\mathrm{a}}$ \\
\hline
\end{tabular}

$P A R$, 光合有效辐射。同列不同小写字母表示样地间差异显著 $(p<0.05)$ 。

$P A R$, photosynthetically active radiation. Different lowercase letters in the same column indicate significant differences among plots $(p<0.05)$.

表2 不同样地芨芨草各功能性状(平均值土标准误差, $n=30$ )

Table 2 Functional characteristics of Achnatherum splendens in different plots (mean $\pm S E, n=30$ )

\begin{tabular}{|c|c|c|c|c|}
\hline \multirow{2}{*}{ 各功能性状 Functional characteristic } & \multicolumn{4}{|c|}{ 样地 Plot } \\
\hline & I & II & III & IV \\
\hline 株高 Plant height (cm) & $130.34 \pm 4.50^{\mathrm{b}}$ & $149.67 \pm 4.62^{\mathrm{a}}$ & $152.00 \pm 5.24^{\mathrm{a}}$ & $95.67 \pm 3.56^{\mathrm{c}}$ \\
\hline 分枝数 Twig number (No. clump $\left.{ }^{-1}\right)$ & $203.33 \pm 8.83^{\mathrm{a}}$ & $173.67 \pm 6.34^{\mathrm{b}}$ & $155.33 \pm 5.90^{\mathrm{b}}$ & $113.33 \pm 4.29^{\mathrm{c}}$ \\
\hline 叶片厚度 Leaf thickness (mm) & $0.145 \pm 0.01^{\mathrm{c}}$ & $0.158 \pm 0.02^{\mathrm{b}}$ & $0.162 \pm 0.02^{\mathrm{b}}$ & $0.166 \pm 0.02^{\mathrm{a}}$ \\
\hline 叶脉直径 Vein diameter (mm) & $0.208 \pm 0.04^{\mathrm{a}}$ & $0.195 \pm 0.03^{\mathrm{b}}$ & $0.184 \pm 0.02^{b}$ & $0.164 \pm 0.02^{\mathrm{c}}$ \\
\hline 叶脉密度 Vein destiny $\left(\mathrm{mm} \cdot \mathrm{mm}^{-1}\right)$ & $1.283 \pm 0.05^{\mathrm{c}}$ & $1.406 \pm 0.04^{\mathrm{b}}$ & $1.439 \pm 0.03^{\mathrm{b}}$ & $1.587 \pm 0.03^{\mathrm{a}}$ \\
\hline$P_{\mathrm{n}}\left(\mu \mathrm{mol} \mathrm{CO} \mathrm{CO}_{2} \cdot \mathrm{m}^{-2} \cdot \mathrm{s}^{-1}\right)$ & $13.20 \pm 0.12^{\mathrm{c}}$ & $13.94 \pm 0.17^{\mathrm{a}}$ & $14.05 \pm 0.18^{\mathrm{a}}$ & $13.87 \pm 0.13^{b}$ \\
\hline$T_{\mathrm{r}}\left(\mathrm{mmol} \mathrm{H} \mathrm{H}_{2} \mathrm{O} \cdot \mathrm{m}^{-2} \cdot \mathrm{s}^{-1}\right)$ & $6.83 \pm 0.11^{\mathrm{a}}$ & $6.67 \pm 0.10^{\mathrm{b}}$ & $6.63 \pm 0.09^{b}$ & $6.35 \pm 0.07^{\mathrm{c}}$ \\
\hline$W U E\left(\mu \mathrm{mol} \mathrm{CO}{ }_{2} \cdot \mathrm{mmol}^{-1} \mathrm{H}_{2} \mathrm{O}\right)$ & $1.93 \pm 0.01^{\mathrm{c}}$ & $2.08 \pm 0.02^{\mathrm{b}}$ & $2.12 \pm 0.02^{\mathrm{b}}$ & $2.18 \pm 0.03^{\mathrm{a}}$ \\
\hline
\end{tabular}

$P_{\mathrm{n}}$, 净光合速率; $T_{\mathrm{r}}$, 蒸腾速率; $W U E$, 水分利用效率。同行不同小写字母表示样地间差异显著 $(p<0.05)$ 。

$P_{\mathrm{n}}$, net photosynthetic rate; $T_{\mathrm{r}}$, transpiration rate. WUE, water use efficiency. Different lowercase letters in the same line indicate significant differences among plots $(p<0.05)$. 


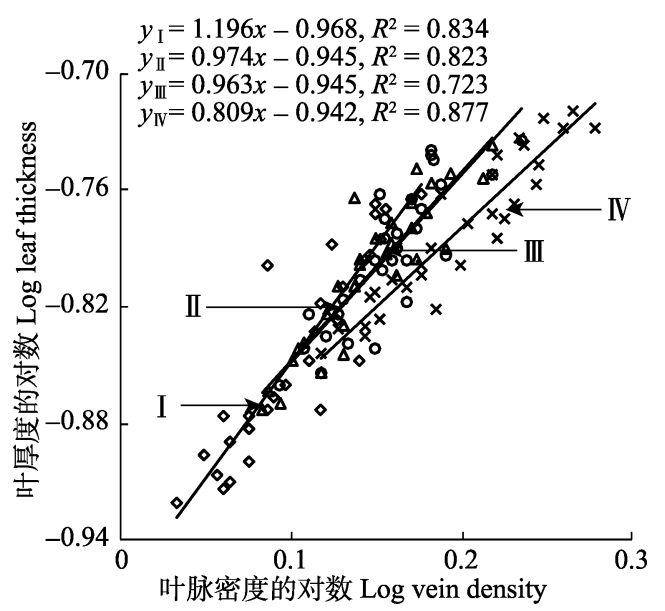

图2 不同土壤水分条件下芨芨草叶片厚度与叶脉密度的关 系。I, 样地I (土壤含水量 $50.07 \%$ ); II, 样地 II (土壤含水量 $38.77 \%$ ); III, 样地III (土壤含水量 $31.5 \%$ ); IV, 样地IV (土壤 含水量 $20.4 \%$ )。

Fig. 2 Relationship between leaf thickness and vein density of Achnatherum splendens among different soil moisture conditions. I, plot I (soil moisture content 50.07\%); II, plot II (soil moisture content $38.77 \%$ ); III, plot III (soil moisture content $31.5 \%$ ); IV, plot IV (soil moisture content 20.4\%).

0.8089 (95\%的置信区间: $C I=0.7644-0.8572)$ 。从 样地I到样地IV SMA斜率依次呈现逐渐减小的趋势, 样地I的SMA 斜率显著大于 $1.0(p<0.05)$, 样地 II和 III的SMA斜率均与 1.0 无显著差异 $(p>0.05)$, 样地 IV的SMA斜率显著小于 $1.0(p<0.05)$ 。表明芨芨草 叶片厚度随着叶脉密度的增加而呈显著增加的趋势, 在不同样地间呈现不同程度的异速生长关系 $(p<$ 0.05), 在样地I芨芨草叶片厚度的增加速率大于叶 脉密度的增加速率; 样地II和III 二者呈近等速生长 关系; 样地IV叶脉密度增加的速度大于叶片厚度增 加的速度, 土壤水分的异质性分布影响了菆菆草叶 片厚度和叶脉密度的资源权衡机制。

\section{4 不同土壤水分条件下湿地植物芨芨草叶片厚 度和叶脉直径的关系}

如图3所示, 不同土壤水分条件下菆芨草叶片 厚度和叶脉直径呈显著的负相关关系 $(p<0.05)$ 。通 过SMA分析得到, 芨芨草在不同样地间的标准主轴 化斜率存在差异，样地㻦 -0.7773 (95\%的置信区间: $C I=-0.7830,-0.7416$ )、样地II为 -0.9566 (95\%的置 信区间: $C I=-0.1012,-0.943$ 1)、样地III为 -1.0354 (95\%的置信区间: $C I=-1.0745,-0.9812$ )、样地IV 为 -1.1165 (95\%的置信区间: $C I=-1.1744,-1.0827$ ), 从样地I到样地IV, SMA斜率的绝对值呈现逐渐增 大的趋势, 样地I的SMA斜率显著大于 $-1.0(p<$

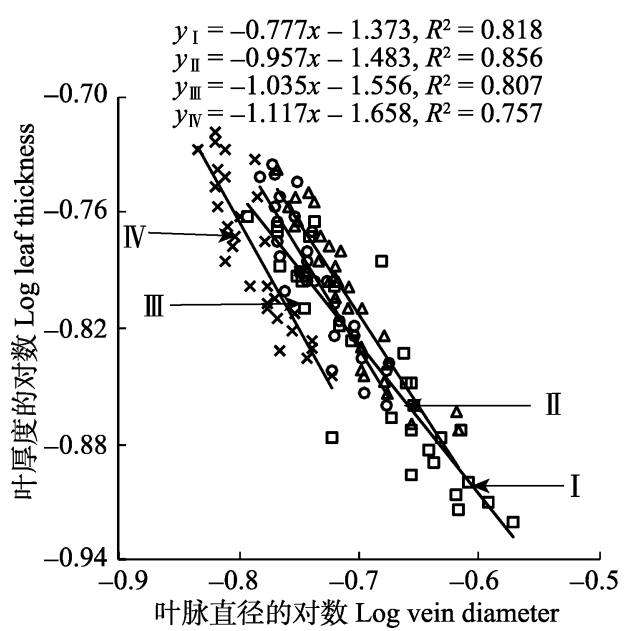

图3 不同土壤水分条件下菠芨草叶片厚度与叶脉直径的关 系。I, 样地I (土壤含水量50.07\%); II, 样地II (土壤含水量 $38.77 \%$ ); III, 样地III (土壤含水量 $31.5 \%$ ); IV, 样地IV (土壤 含水量 $20.4 \%$ )。

Fig. 3 Relationship between leaf thickness and vein diameter of Achnatherum splendens among different soil moisture conditions. I, plot I (soil moisture content 50.07\%); II, plot II (soil moisture content $38.77 \%$ ); III, plot III (soil moisture content $31.5 \%$ ); IV, plot IV (soil moisture content $20.4 \%$ ).

0.05), 样地II和III的SMA斜率均与-1.0无显著差异 $(p>0.05)$, 样地IV的SMA 斜率显著小于 $-1.0(p<$ 0.05)。表明芨芨草随着叶片厚度的逐渐增大，叶脉 直径呈相反的变化趋势，二者在不同样地呈显著的 权衡生长关系 $(p<0.05)$, 且在样地I芨菆草叶片厚度 减小的速度小于叶脉直径减小的速度; 样地II和III 叶片厚度增加的速度接近叶脉直径减小的速度; 样 地IV叶片厚度增加的速度大于叶脉直径减小的速 度, 不同生境中土壤含水量的差异加剧了叶片厚度 与叶脉直径的资源权衡分配机制。

\section{3 讨论}

叶片水力阻力是植物应对环境水分和光照胁 迫的主要限制因子之一(Scoffoni et al., 2011), 被视 作植物水分传输过程中的一个水力瓶颈，是应对灾 难性水力失调的安全阀门(Villagra et al., 2013)。分 析叶片厚度与叶脉密度、叶脉直径的异速生长关系 对认识叶片适应不同水分环境具有重要作用。为了 缓解生理活动中的水分胁迫, 植物往往在叶片水分 防御和输导的构造上进行资源权衡投资(许洺山等, 2015), 这影响着植物叶片的形态构建及代谢活动 中的碳分配(Moles et al., 2014)。本研究发现, 在不 同土壤水分条件下, 菆菆草叶片厚度与叶脉密度、 叶脉直径间存在不同的生长关系 $(p<0.05)$, 在样地 
I和IV, SMA斜率的绝对值均与 1.0 存在显著差异 $(p<$ 0.05 ), 在样地II和III, SMA 斜率的绝对值与 1.0 无显 著差异 $(p>0.05)$ (图2, 图3)。不同土壤水分样地芨芨 草叶性状的异速生长关系及敏感性差异, 表明湿地 植物在维持水力安全和碳利用效率间存在着潜在的 权衡机制，对于理解和预测不同环境下植物的存 活、生长、繁衍和分布具有重要的意义。

\section{1 高土壤含水量条件下菠芨草叶片厚度和叶脉 性状之间的生长关系}

植物的水分适应能力是长期适应环境的一种 复杂的遗传特性, 与植物形态结构、内部生理生化 活动和外界条件等密切相关(李荣等, 2015), 植物会 通过调整光合构件生长率、权衡资源分配等长期的 表型可塑性来增加异质性资源的受食效率(侯兆疆 等, 2014)。研究区属于河西走廊温带干旱区的天然 湿地生态系统, 土壤水和地下水可能是芨芨草的主 要利用水源(吴华武等, 2015)。在土壤水分较高的样 地I, 湿地群落密度和高度最大(表1), 光竞争成为植 被生长中面对的首要问题, 群落冠层内的芨芨草获 得的 $P A R$ 更少(表1, 表2), 为了保证自身的生理活性 和体内水分的动态平衡, 芨芨草增加分枝数以获取 更多的光资源(表2), 并进行叶内生物量的权衡分配 和叶片构型塑造的调整, 形成了增加叶脉直径, 减 小叶片厚度和叶脉密度的资源分配机制, 形成了叶 片厚度生长速度显著大于叶脉密度的生长速度, 而 小于叶脉直径减小速率的异速生长格局 $(p<0.05$, 图 2 , 图3)。主要原因有: (1)该样地中芨芨草株高较小(表 2 ), 为了保证有限光环境中最大的碳同化速率和光 合能力, 需加强叶脉直径的营养物质投资, 以较强 的机械支撑强度支持更大的展叶面积, 伴随着叶片 厚度的减小(表2), 薄叶片能够使 $\mathrm{CO}_{2}$ 和水分等从气 孔到达叶绿体的路径缩短, 有利于光的透过和光能 的吸收(Hultine \& Marshall, 2000; 宋璐璐等, 2012), 进而提高了植物的生存适合度和群落竞争力; (2)样 地中土壤含水量较高时植物木质部导水速率较高 (周洪华等和李卫红, 2015), 叶片水分充足, 较小的 叶脉密度也能满足较高蒸腾速率 $\left(T_{\mathrm{r}}\right)$ 的水分需求 (表 $2)$, 芨菆草以有限的生物量增加叶脉直径来提高水 分运输的量与速率, 消除了叶脉密度变小的弊端, 同时通过减小叶片厚度减少了水分输送阻力, 以确 保植物保持自身碳、矿物质以及水分的收支平衡, 从而保证了芨芨草种群的顺利繁衍和更新。

\section{2 低土壤含水量条件下芨芨草叶片厚度和叶脉 性状的生长关系}

植物叶脉系统水分运输的有效性与抗栓塞的 安全性有一定的动态权衡, 这种权衡性可能是水分 有效运输的最佳值(Scoffoni et al., 2015)。单位叶面 积上木质部通道较多的植物, 其叶片的流体传导性 和叶水力导度更强(Sack \& Scoffoni, 2013), 有利于 输送水分、养分和光合产物(Brodribb et al., 2007; McKown et al., 2010)。湿地群落土壤含水量最小的 样地 IV, 群落高度和密度显著减小(表1), 芨菆草接 收到的 $P A R$ 最大 (表1), 随着土壤水分潜在蒸散的增 大, 伴随着土壤电导率最大(表1), 盐胁迫引起土壤 水势降低, 诱导植物发生水分胁迫(Kang \& Zhang, 2004; 李荣等, 2015), 与典型盐生植物相比, 芨芨 草并非喜盐植物(张明娟等, 2012), 在水盐胁迫为主 的条件下，往往易造成植物木质部导管栓塞(Sperry， 2000; 龚容和高琼, 2015), 芨苃草水分输导的有效 性和安全性显著下降, 促使芨芨草进行叶性状间的 资源权衡分配，并构建与其相适应的叶片几何构型 以缓解植物体水分胁迫, 菆芝草趋向于叶片厚度的 增加速率小于叶脉密度增加速率、大于叶脉直径减 小速率的异速生长模式 $(p<0.05$, 图2, 图3), 主要 原因有: (1)面对该生境中较高的 $P A R$, 芨芨草选择 增加叶片厚度的资源投资, 既能通过增大水蒸气进 出表皮阻力降低叶片蒸腾速率(表2), 也能减少植物 内部的水分散失(Ogburn \& Edwards, 2012), 且厚叶 片较长的栅栏细胞和更多的细胞层数能减少光辐射 的穿透量, 又避免了强光对叶片的伤害(于鸿䒯等, 2014); (2)该生境中土壤含水量较样地 $\mathrm{I}$ 显著减少 $59.26 \%$, 为了降低水分胁迫下叶片水力功能紊乱和 脱水的风险，芨夰草增加叶脉密度使得水分运输的 路径增多, 叶片可以绕过气蚀化的木质部把水分运 输到蒸腾部位的细胞(Sack et al., 2013), 且植物小 径叶脉的导管壁厚(Givnish, 1987), 芨芨草减小叶 脉直径的资源分配，降低了导管坍塌和破坏的几率， 大大提高了水分运输的安全性和水分利用效率(表 2), 与Nardini (2012)等关于“直径小且数量多的叶脉 水分运输效率高”一致, 体现了菆芨草通过环境因 子对植物水力结构的影响和驯化, 减少了营养的 “流失”, 并提高应对水分胁迫、维持光合生产和自 我保护的调控能力。

叶形态和生理特性在植物碳同化、水分关系和 
能量平衡等方面有重要作用, 决定了叶片功能属性 在不同环境条件下的差异 (Wright et al., 2004; Poorter \& Bongers, 2006)。芨芨草喜生于地下水埋深 $1.5 \mathrm{~m}$ 左右的盐碱滩沙质土壤上(张明娟等, 2012), 在地下水埋深为 $1.0-2.0 \mathrm{~m}$, 且土壤水分适中的样地 II和样地III, 湿地群落环境中群落高度、密度和地表 土壤水分、理化过程和强度均处于干旱到湿润的过 渡区，种间对空间、光和土壤水分、养分的竞争有 所减弱(表1), 比较适宜芨芨草的生长, 芨芨草 $P_{\mathrm{n}}$ 和 株高最大(表2), 使其能利用较为充足的物质和能量 资源进行高效的光合作用和物质运输, 芨芨草选择 了折中水分输送和存储结构的稳步投资, 形成了叶 片厚度与叶脉密度、叶脉直径均近等速生长的格局 $(p<0.05$ 、图2-3), 也是叶片厚度由薄到厚、叶脉密 度由小到大、叶脉直径由粗到细转变的区域, 即在 该环境中保持较高的光合能力和竞争力, 保证了植 株的顺利生长和种群的繁衍更新, 从而成为湿地群 落中的优势种。

\section{4 结论}

生长在土壤含水量丰富的近河岸湿地群落样 地中的芨芨草叶片厚度增长的速度快于叶脉密度增 加的速度, 从而导致产生了较薄的叶片, 叶脉粗, 数量少, 倾向于采取高蒸腾速率的开拓性策略, 而 远离河岸的芨芨草叶脉密度增加的速度大于叶片厚 度增加的速度, 具有较厚的叶片, 叶脉细, 数量多, 选择了以降低水分消耗为主的保守性策略, 在不同 水分环境下形成了湿地植物叶片水分传输通道表型 可塑性机制。

基金项目 国家自然科学基金(41461013和91125014)。

\section{参考文献}

Berlyn GP, Miksche JP (1976). Botanical Microtechnique and Cytochemistry. Iowa State University Press, Ames.

Blonder B, Violle C, Bentley LP, Enquist BJ (2010). Venation networks and the origin of the leaf economics spectrum. Ecology Letters, 14, 91-100.

Brodribb TJ, Jordan GJ (2011). Water supply and demand remain balanced during leaf acclimation of Nothofagus cunninghamii trees. New Phytologist, 192, 437-448.

Brodribb TJ, Feild TS, Jordan GJ (2007). Leaf maximum photosynthetic rate and venation are linked by hydraulics. Plant Physiology, 144, 1890-1898.

Domínguez MT, Aponte C, Perez-Ramos IM (2012). Relation- ships between leaf morphological traits, nutrient concentrations and isotopic signatures for Mediterranean woody plant species and communities. Plant and Soil, 357, 407424.

Falster DS, Warton DI, Wright IJ (2012). User's Guide to SMATR: Standardised Major Axis Tests \& Routines. Version 2.0. http://www.bio.mq.edu. au/ecology/SMATR/. Cited: 2014-03-11.

Gago J, Douthe C, Florez-Sarasa I, Escalona JM, Galmes J, Fernie AR, Flexas J, Medrano H (2014). Opportunities for improving leaf water use efficiency under climate change conditions. Plant Science, 226, 108-119.

Givnish TJ (1987). Comparative studies of leaf form: Assessing the relative roles of selective pressures and phylogenetic constraints. The New Phytologist, 106, 131-160.

Gong R, Gao Q (2015). Research progress in the effects of leaf hydraulic characteristics on plant physiological functions. Chinese Journal of Plant Ecology, 39, 300-308. (in English with Chinese abstract) [龚容, 高琼 (2015). 叶片结 构的水力学特性对植物生理功能影响的研究进展. 植 物生态学报, 39, 300-308.]

Harvey PH, Pagel MD (1991). The Comparative Method in Evolutionary Biology. Oxford University Press, Oxford, UK.

Han L, Zhao CZ, Xu T, Feng W, Duan BB, Zheng HL (2016). Trade-off between leaf size and vein density of Achnatherum splendens in Zhangye wetland. Chinese Journal of Plant Ecology, 40, 788-797. (in Chinese with English abstract) [ 韩玲, 赵成章, 徐婷, 冯威, 段贝贝, 郑慧玲 (2016). 张掖湿地菆芨草叶大小和叶脉密度的权衡关系. 植物生态学报, 40, 788-797.]

Hou ZJ, Zhao CZ, Li Y, Zhang Q, Ma XL (2014). Trade-off between height and branch numbers in Stellera chamaejasme on slopes of different aspects in a degraded alpine grassland. Chinese Journal of Plant Ecology, 38, 281-288. (in English with Chinese abstract) [侯兆疆, 赵成章, 李钰, 张 茜, 马小丽 (2014). 不同坡向高寒退化草地狼毒株高和 枝条数的权衡关系. 植物生态学报, 38, 281-288.]

Hultine KR, Marshall JD (2000). Altitude trends in conifer leaf morphology and stable carbon isotope composition. Oecologia, $123,32-40$.

Kang SZ, Zhang JH (2004). Controlled alternate partial rootzone irrigation: Its physiological consequences and impact on water use efficiency. Journal of Experimental Botany, 55, 2437-2446.

Li R, Jiang ZM, Zhang SX, Cai J (2015). A review of new research progress on the vulnerability of xylem embolism of woody plants. Chinese Journal of Plant Ecology, 39, 838848. (in English with Chinese abstract) [李荣, 姜在民, 张 硕新, 蔡靖 (2015). 木本植物木质部栓塞脆弱性研究新 进展. 植物生态学报, 39, 838-848.]

Matthew-Ogburn R, Edwards EJ (2013). Repeated origin of three-dimensional leaf venation releases constraints on the

www.plant-ecology.com 
evolution of succulence in plants. Current Biology, 23, $722-726$.

McKown AD, Cochard H, Sack L (2010). Decoding leaf hydraulics with a spatially explicit model: Principles of venation architecture and implications for its evolution. The American Naturalist, 175, 447-460.

Moles AT, Perkins SE, Laffan SW (2014). Which is a better predictor of plant traits: Temperature or precipitation? Journal of Vegetation Science, 25, 1167-1180.

Nardini A, Luglio J (2014). Leaf hydraulic capacity and drought vulnerability: Possible trade-offs and correlations with climate across three major biomes. Functional Ecology, 28, 810-818.

Nardini A, Pedà G, La Rocca N (2012). Trade-offs between leaf hydraulic capacity and drought vulnerability: Morphoanatomical bases, carbon costs and ecological consequences. New Phytologist, 196, 788-798.

Nardini A, Raimondo F, LoGullo MA (2010). Leaf miners help us understand leaf hydraulic design. Plant, Cell \& Environment, 33, 1091-1100.

Ogburn RM, Edwards EJ (2012). Quantifying succulence: A rapid, physiologically meaningful metric of plant water storage. Plant, Cell \& Environment, 35, 1533-1542.

Pausas JG, Austin MP (2001). Patterns of plant species richness in relation to different environments: An appraisal. Journal of Vegetation Science, 12, 153-166.

Perez-Ramos IM, Roumet C, Cruz P (2012). Evidence for a plant community economics spectrum driven by nutrient and water limitations in a Mediterranean rangeland of southern France. Journal of Ecology, 100, 1315-1327.

Poorter L, Bongers F (2006). Leaf traits are good predictors of plant performance across 53 rain forest species. Ecology, 87, 1733-1743.

Quan XK, Wang CK (2015). Comparison of foliar water use efficiency among 17 provenances of Larix gmelinii in the Mao'ershan area. Chinese Journal of Plant Ecology, 39, 352-361. (in English with Chinese abstract) [全先奎, 王 传宽 (2015). 帽儿山17个种源落叶松针叶的水分利用 效率比较. 植物生态学报, 39, 352-361.]

Ren QJ, Li HL, Bu HY (2015). Comparison of physiological and leaf morphological traits for photosynthesis of the 51 plant species in the Maqu alpine swamp meadow. Chinese Journal of Plant Ecology, 39, 593-603. (in English with Chinese abstract) [任青吉, 李宏林, 卜海燕 (2015). 玛 曲高寒沼泽化草甸 51 种植物光合生理和叶片形态特征 的比较. 植物生态学报, 39, 593-603.]

Reich PB, Cornelissen H (2014). The world-wide 'fast-slow' plant economics spectrum: A traits manifesto. Journal of Ecology, 102, 275-301.

Sack L, Scoffoni C, McKown AD (2012). Developmentally based scaling of leaf venation architecture explains global ecological patterns. Nature Communications, 3, 837.
Sack L, Scoffoni C (2013). Leaf venation: Structure, function, development, evolution, ecology and applications in the past, present and future. New Phytologist, 198, 983-1000.

Sack L, Scoffoni C, John GP, Poorter H, Mason CM, Mendez-Alonzo R, Donovan LA (2013). How do leaf veins influence the worldwide leaf economic spectrum? Review and synthesis. Journal of Experimental Botany, 64, 40534080.

Scoffoni C, Rawls M, McKown A, Cochard H, Sack L (2011). Decline of leaf hydraulic conductance with dehydration: Relationship to leaf size and venation architecture. Plant Physiology, 156, 832-843.

Scoffoni C, Kunkle J, Pasquet-Kok J, Vuong C, Patel AJ, Montgomery RA, Givnish TJ, Sack L (2015). Light induced plasticity in leaf hydraulics, venation, anatomy, and gas exchange in ecologically diverse Hawaiian lobeliads. New Phytologist, 207, 43-58.

Silvertown J, Charlesworth D (2001). Introduction to Plant Population Biology. Blackwell, London.

Song LL, Fan JW, Wu SH, Zhong HP, Wang N (2012). Response characteristics of leaf traits of common species along an altitudinal gradient in Hongchiba Grassland, Chongqing. Acta Ecologica Sinica, 32, 2759-2767. (in English with Chinese abstract) [宋璐路，禁江文，吴绍洪， 钟华平, 王宁 (2012). 红池坝草地常见物种叶片性状沿 海拔梯度的响应特征. 生态学报, 32, 2759-2767.]

Sperry JS (2000). Hydraulic constraints on plant gas exchange. Agricultural and Forest Meteorology, 104, 13-23.

Villagra M, Campanello PI, Bucci SJ, Goldstein G (2013). Functional relationships between leaf hydraulics and leaf economic traits in response to nutrient addition in subtropical tree species. Tree Physiology, 33, 1308-1318.

Wang RL, Yu GR, He NP, Wang QF, Zhao N, Xu ZW (2015). Latitudinal patterns and influencing factors of leaf functional traits in Chinese forest ecosystems. Acta Geographica Sinica, 70, 1735-1746. (in English with Chinese abstract) [王瑞丽, 于贵瑞, 何念鹏, 王秋风, 赵宁, 徐志伟 (2015). 中国森林叶片功能属性的纬度格局及其影响因 素. 地理学报, 70, 1735-1746.]

Warton DI, Weber NC (2002). Common slope tests for bivariate errors-in-variables models. Biometrical Journal, 44, 161-174.

Warton DI, Wright IJ, Falster DS, Westoby M (2006). Bivariate line-fitting methods for allometry. Biological Reviews, 81, 259-291.

Wang J, Cheng JM, Wan HE, Fang F (2004). Study on soil moisture characteristics and water use efficiency of Achnatherum splendens grassland in Loess Plateau. Arid Meteorology, 22, 51-55.

Wilson PJ, Thompson K, Hodgson JG (1999). Specific leaf area and leaf dry matter content as alternative predictors of plant strategies. New Phytologist, 143, 155-162.

Wright IJ, Reich PB, Westoby M, Ackerly DD, Baruch Z, 
Bongers F, Cavender-Bares J, Chapin T, Cornelissen JH, Diemer M, Flexas J, Garnier E, Groom PK, Gulias J, Hikosaka K, Lamont BB, Lee T, Lee W, Lusk C, Midgley JJ, Navas ML, Niinemets U, Oleksyn J, Osada N, Poorter H, Poot P, Prior L, Pyankov VI, Roumet C, Thomas SC, Tjoelker MG, Veneklaas EJ, Villar R (2004). The worldwide leaf economics spectrum. Nature, 428, 821-827.

Wu HW, Li XY, Jing ZY, Li J, Zheng XR, Zhao DZ (2015). Variations in water use for Achnatherum splendens in Lake Qinghai watershed based on $\delta \mathrm{D}$ and $\delta^{18} \mathrm{O}$. Acta Ecologica Sinica, 35, 8174-8183. (in English with Chinese abstract) [吴华武, 李小雁, 蒋志云, 李静, 郑肖然, 赵 殿智 (2015). 基于 $\delta \mathrm{D} 、 \delta^{18} \mathrm{O}$ 的青海湖流域芨芨草水分利 用来源变化研究. 生态学报, 35, 8174-8183.]

Xu MS, Huang HX, Shi QR, Yang XD, Zhou LL, Zhao YT, Zhang QQ, Yan ER (2015). Responses of soil water content to change in plant functional traits in evergreen broadleaved forests in eastern Zhejiang Province. Chinese Journal of Plant Ecology, 39, 857-866. (in English with Chinese abstract) [许洺山, 黄海侠, 史青茹, 杨晓东, 周 刘丽, 赵延涛, 张晴晴, 阎恩荣 (2015). 浙东常绿阔叶 林植物功能性状对土壤含水量变化的响应. 植物生态 学报, 39, 857-866.]

Xu T, Zhao CZ, Duan BB, Han L, Zhen HL, Feng W (2016). Slope-related variations of different levels of vein density and leaf size in Robinia pseudoacacia in the northern mountains of Lanzhou. Chinese Journal of Ecology, 35, 41-47. (in English with Chinese abstract) [徐婷, 赵成章, 段贝贝, 韩玲, 郑慧玲, 冯威 (2016). 兰州北山刺槐不 同等级叶脉密度与叶大小关系的坡向差异性. 生态学 杂志, 35, 41-47.]

Yu HY, Chen YT, Xu ZZ, Zhou GS (2014). Analysis of relationships among leaf functional traits and economics spectrum of plant species in the desert steppe of Nei Mongol. Chinese Journal of Plant Ecology, 38, 1029-1040. (in
English with Chinese abstract) [于鸿莹，陈芗婷，许振柱， 周广胜 (2014). 内蒙古荒漠草原植物叶片功能性状关 系及其经济谱分析. 植物生态学报, 38, 1029-1040.]

Zhang SB, Guan ZJ, Sun M, Zhang JJ, Cao KF, Hu H (2012). Evolutionary association of stomatal traits with leaf vein density in Paphiopedilum, Orchidaceae. PLOS ONE, 7: e40080. doi: 10.1371/journal.pone.0040080.

Zhang ZL, Liu GD, Zhang FC, Zheng CX, Kang YH (2014). Research progress of plant leaf hydraulic conductivity. Chinese Journal of Ecology, 33, 1663-1670. (in English with Chinese abstract) [张志亮, 刘国东, 张富仓, 郑彩 霞, 康银红 (2014). 植物叶片导水率的研究进展. 生态 学杂志, 33, 1663-1670.]

Zhang MJ, Liu MS, Xu C, Chi T, Hong C (2012). Spatial pattern responses of Achnatherum splendens to environmental stress in different density levels. Acta Ecologica Sinica, 32, 595-604. (in English with Chinese abstract) [张明娟, 刘茂松, 徐驰, 池婷, 洪超 (2012). 不同密度条件下茬 芨草空间格局对环境胁迫的响应. 生态学报，32，595604.]

Zhang YQ, Liang CZ, Wang W, Wang LX, Peng JT, Yan JC, Jia JC (2010). Soil salinity and Achnatherum splendens distribution. Chinese Journal of Ecology, 29, 2438-2443. (in English with Chinese abstract) [张雅琼, 梁存柱, 王 炜, 王立新, 彭江涛, 间建成, 贾成朕 (2010). 菆菆草 群落土壤盐分特征. 生态学杂志, 29, 2438-2443.]

Zhou HH, Li WH (2015). Responses and adaptation of xylem hydraulic conductivity to salt stress in Populus euphratica. Chinese Journal of Plant Ecology, 39, 81-91. (in English with Chinese abstract) [周洪华, 李卫红 (2015). 胡杨木 质部水分传导对盐胁迫的响应与适应. 植物生态学报, 39, 81-91.]

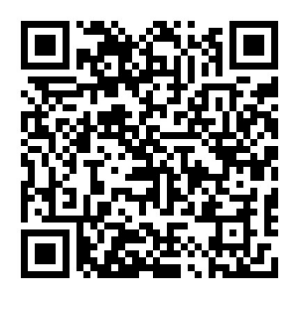

www.plant-ecology.com 